\title{
Vertical Handover Study on 4G Category vs. 5G Category for 3GPP Generation Mobile Systems and Non-3GPP Wireless Networks
}

\author{
Dr Omar Khattab \\ Department of Computer Networks \& Communications \\ Computer Sciences \& Information Technology, King Faisal University \\ Kingdom of Saudi Arabia \\ okhattab@kfu.edu.sa
}

\begin{abstract}
Nowadays, wireless communication technologies have become an integral part of people's daily life and businesses all over the world. Due to the rapid increase in the number of the Mobile Users (MUs) who demand the service of communicating via wireless access networks, the wireless communication technologies have evolved from the first generation to the fifth generation. The Next Generation Wireless Systems (NGWSs) consists of heterogeneous wireless access networks of 3GPP generation mobile Systems (e.g., UMTS, LTE) and non-3GPP wireless networks (e.g., WiFi, WiMAX), where MUs can access these technologies and services using a single device. This paper presents a study for Vertical Handover (VHO) approaches of 3GPP and non-3GPP proposed in the literature and classifies them into two categories for which their characteristics are discussed.
\end{abstract}

Keywords: Vertical Handover; Mobile Systems; Wireless Networks; 4G; 5 G.

\section{Introduction}

The rapid evolutions in broadband wireless communication technologies and the growing Mobile Users' demand (MUs) for communication services anywhere, anytime are driving an evolution toward the seamless integration between different Radio Access Technology (RATs) in heterogeneous wireless communication technologies to provide the best connected services to the MU constantly [1]. The benefits of heterogeneous wireless communication technologies are many and varied. These include: flexibility, reducing cost, simplifying the operation and maintenance, rapid deployment of services and applications, new services, high data transmission, customisation, support multimedia services at lower cost of transmission, the mobility of the sessions and the possibility to transfer the context [1].

The Next Generation Wireless Systems (NGWSs) consists of heterogeneous wireless communication technologies of 3GPP and non-3GPP, where MUs can access these technologies and services using a single device. This device is equipped with multiple radio interfaces include devices capable of supporting multiple RATs by incorporating several interface cards and appropriate software for switching between multiple access systems (Vertical Handover (VHO)). 
Dr Omar Khattab; 3GPP Generation Mobile Systems and Non-3GPP Wireless Networks: A New Class of Octagon-Cell Interconnected Networks, Transactions on Networks and Communications, Volume 6 No. 2, April (2018); pp: 1-9

This paper presents background information on heterogeneous wireless communication technologies. Then, it overviews VHO approaches of 3GPP and non-3GPP proposed in the literature and classifies them into two categories for which their characteristics are discussed.

The rest of the paper is organized as follows: In section 2, background information on heterogeneous wireless communication technologies is presented. In section 3, classifications for VHO approaches of 3GPP and non-3GPP are presented. In section 4, a comparison for VHO approaches of 3GPP and non-3GPP is presented and finally, section 5 concludes the paper.

\section{Background on Heterogeneous Wireless Communication Technologies}

In this section, background information on heterogeneous wireless communication technologies is presented to answer the following questions: how have wireless communication technologies evolved? What are heterogeneous wireless communication technologies? Who needs heterogeneous wireless communication technologies? Why are heterogeneous wireless communication technologies necessary? and finally, what is the handover management within heterogeneous wireless communication technologies.

\section{How Have wireless communication technologies Evolved?}

During the last few years, telecommunication authorities were busy while working out how to emerge to the next generation of wireless technology environment which was motivated by the growing demand for advanced telecommunication services which require wider spectrum and higher QoS [2]. Besides, the telecommunication industry experts are required to develop an interoperability strategy for new mobile wireless systems which can satisfy MUs' demands of telecommunication systems [2]. This section presents a background of the main wireless communication technologies, as shown in Table 1.

\subsection{GSM}

GSM is a $2 \mathrm{G}$ mobile system which is the first one to specify digital modulation and network level architectures and services, the first important set of Radio Frequency (RF) for GSM standard started at $1900 \mathrm{MHz}$ [3]. GSM was first introduced in Europe in 1991 and today is one of the most popular digital mobile telecommunications systems widely used over the world [3]. Due to the increase of the number and the requirement of GSM subscriber the GSM is still an attractive area for research in the field of mobile telecommunication [3-5].

\subsection{UMTS}

2G like GSM were originally designed for efficient delivery of voice services. 3G systems like UMTS were designed from the beginning for mobile voice and data users [6]. Therefore, UMTS is the evolution of GSM system and General Radio Packet Service (GPRS) developed by Third Generation Partnership Project (3GPP) to increase the support for some features such as data rate in radio interface and the compatibility for the two services domains: Packet Switched (PS) and Circuit Switched (CS) data transmission [7]. Some of the most common keys drive of this type of UMTS access technology [8]:

- Growth in the market for fixed networked multimedia services.

- Increasing demand for rapid and remote access to information.

- E-Commerce and transaction based applications. 


\subsection{Wi-Fi}

The Wi-Fi (IEEE 802.11) is wireless networks designed to provide broadband for Wireless Local Area Network (WLAN) where the MUs use the mobile devices (e.g., mobiles and laptops) to access the internet in small geographic area such as university's buildings, airports and railway stations. Over $97 \%$ of laptops today come with Wi-Fi as a standard feature and an increasing number of handhelds and Consumer Electronics (CEs) devices are adding Wi-Fi capabilities [9] as Wi-Fi technology in conformance with IEEE 802.11 are growing every year [28, thesis]. The initial standard IEEE 802.11, which came in 1997, had a data rate of $1 \mathrm{Mbps}$ [10]. By year 1999 this was changed; 802.11a (54 Mbps at wider frequency band), 802.11b (11 Mbps, same frequency band but a different modulation technique) and 802.11g (using modulation technique of 802.11a but frequency band of 802.1lb) [10]. During the period between 19902000, the IEEE committee, which had already created wired LAN standards (802.3 Ethernet), started processing wireless LAN standard [10]. As Ethernet was dominant at that time, the committee decided to make wireless standard 802.11 compatible with Ethernet above data link layer; however, it was different from Ethernet in link layer and physical layer due to various issues faced the wireless communication [10].

\section{$2.4 \quad 4 G$}

Growing demand for new applications required to be supported by new mobile systems such as Voice over Internet Protocol (VoIP), video conference, Push to-talk-over Cellular (PoC), multimedia messaging, multiplayer games, Virtual Private Networks (VPNs), web browsing, email access, audio and video Streaming, content download of ring tones, video clips and File Transfer Protocol (FTP) [11]. These applications require higher throughput, wider bandwidth, smaller delay and innovative transmission methods which will give higher spectral efficiency and good quality [2]. Therefore, WiMAX and LTE wireless communication technologies are considered as candidates to achieve the $4 G$ requirements announced by International Telecommunication Radio Communication Sector (ITU-R) which is known as International Mobile Telecommunication-Advanced (IMT-Advanced) [2]. Figure 1 shows the geographical locations of the deployment of $2 \mathrm{G}, 3 \mathrm{G}$ and $4 \mathrm{G}$.

\subsubsection{WiMAX}

WiMAX (IEEE 802.16) is a telecommunication mobile system designed to provide high speed broadband wireless access which is a probable replacement candidate for mobile system (e.g., GSM) or can be used as an overlay to enhance capacity [12]. There are many versions of WiMAX (IEEE 802.16) standards. The IEEE 802.16d (802.16-2004) provides fixed WiMAX network while IEEE 802.16e (802.16-2005) is an amendment to 802.16-2004 and it is directed to support for mobility; therefore, also known as "Mobile WiMAX" [12]. The WiMAX revision IEEE 802.16m expected to offer peak rates of at least 1 Gbps fixed speed and $100 \mathrm{Mbps}$ to MUs [13].

\subsubsection{LTE}

3GPP's LTE standard evolved from the high speed packet access cellular standards. LTE is a telecommunication mobile system designed to provide higher data rate, higher throughput and lower airinterface latency compared with $2 \mathrm{G}$ and $3 \mathrm{G}$ systems [14]. This higher performance makes it possible to enhance the broadband data on demanding applications beyond web browsing and voice which require higher data rate and stricter QoS constraints such as video service [14]. 


\section{$2.5 \quad 5 G$}

$5 \mathrm{G}$ is defined as upcoming mobile system beyond $4 \mathrm{G}$ (B4G) which provides substantial features compared to the current mobile systems $[15,16]$ :

- Better coverage area.

- Higher data rate (around $1 \mathrm{Gbps})$.

- Lower battery consumption.

- Higher security.

- Better spectral efficiency and Energy efficiency.

- Availability of Artificial Intelligence inspired applications.

- Not harmful for human health.

- Economic services due to low deployment cost.

- It has been concluded in [16] that "the final success of $5 G$ will depend upon when it is fully implemented and the new services and contents made available to MUs". Figure 2 shows the geographical locations of the deployment of 2G, 3G, 4G LTE and 5G.

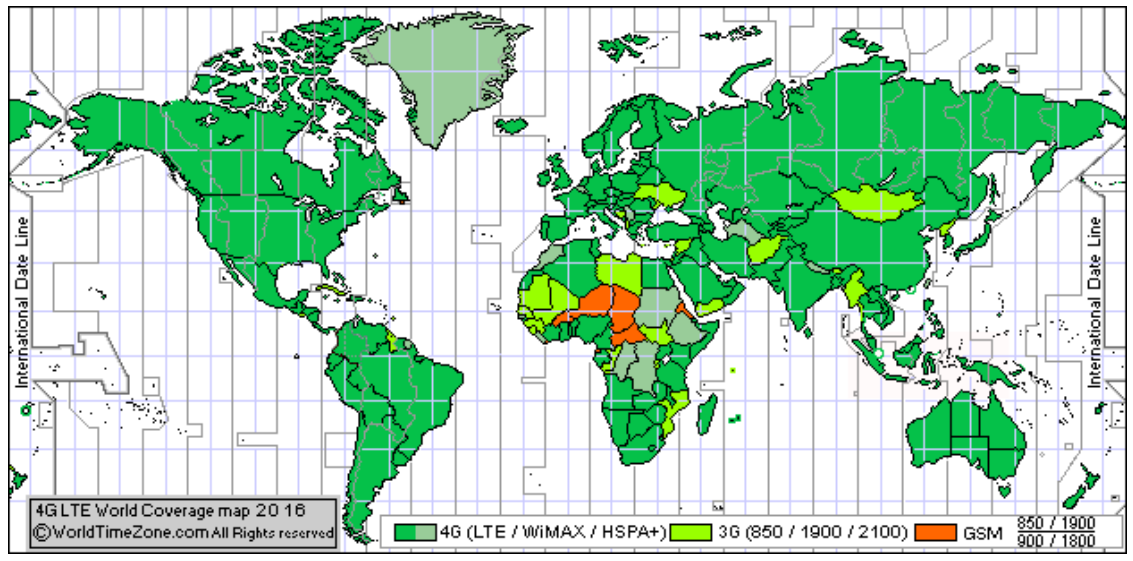

Figure 1. 2G, 3G and 4G world coverage map [17]

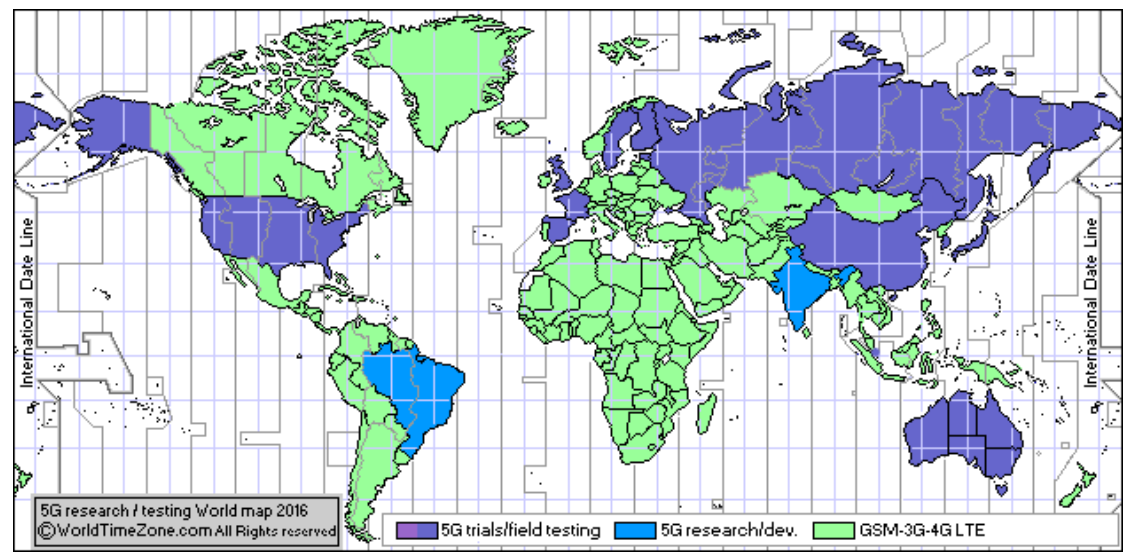

Figure 2. 2G, 3G, 4G LTE and 5G world coverage map [18] 
Table 1. Advantages and disadvantages for UMTS, Wi-Fi, WiMAX, LTE and 5G [1, 12, 14-16, 19-27]

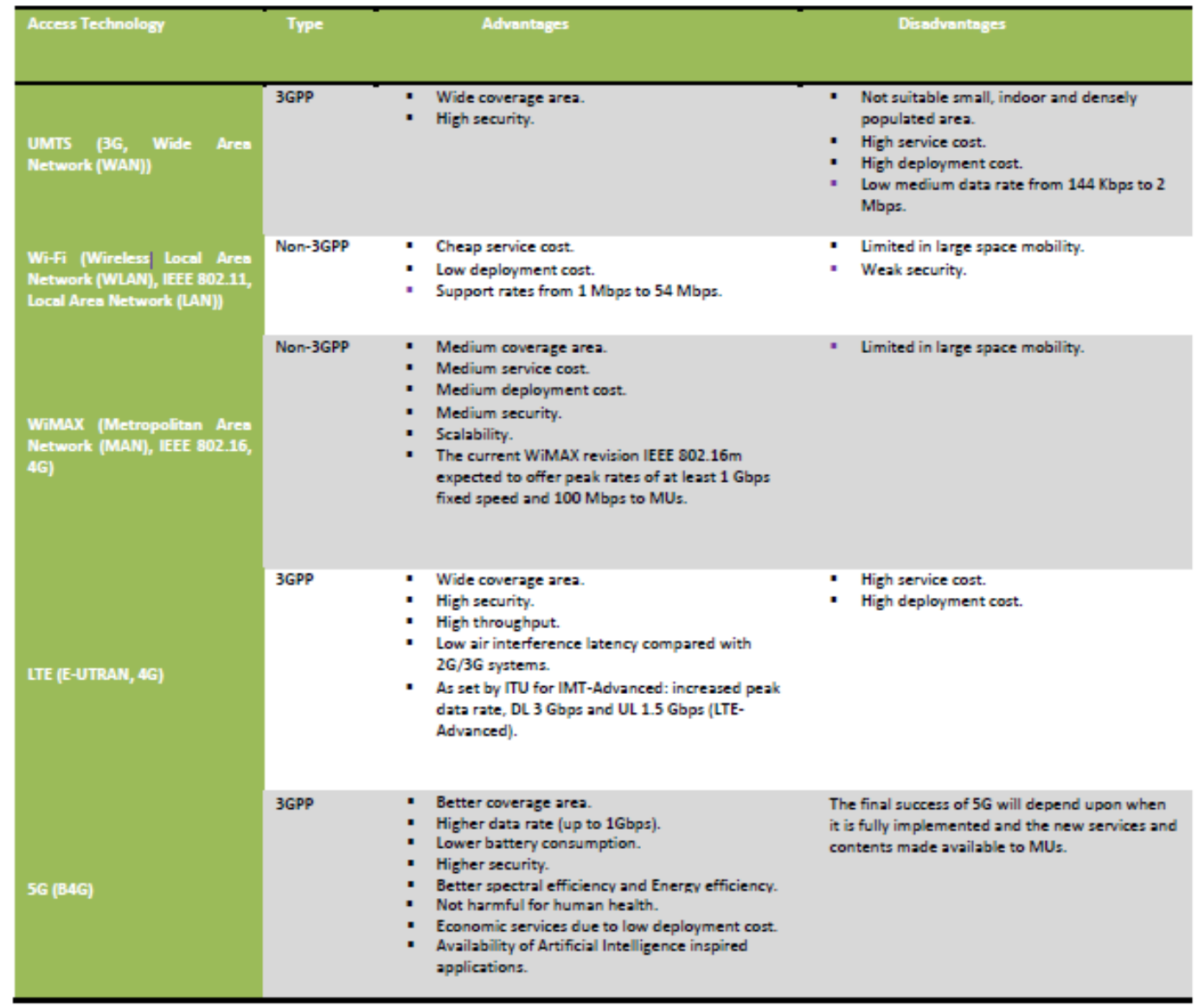

\section{What are heterogeneous wireless communication technologies?}

The growing demand for services (e.g., web browsing, file downloading and e-mail) from MUs anywhere, anytime is on the increase regardless of the technological constraints which are associated with different types of RATs such as UMTS, WiMAX and LTE, besides, there is no single RAT is able to satisfy the requirements for all different wireless communications scenarios. Therefore, the telecommunication operators are required to develop an interoperability strategy for these different types of existing networks to get the best connection anywhere, anytime between heterogeneous wireless communication technologies [28].

\section{Who needs heterogeneous wireless communication technologies?}

There are two main parties that need heterogeneous wireless communication technologies; the first one is the operator and the second is the MUs. The operators always seek to improve the final user experience and optimum use of the network by making a transition from the source network to target network as transparent as possible. The thing which will be reflected positively on operators to get more subscribers (users' loyalty) and more profit eventually; this is shown in Fig. 3. On the

other side, the MUs need to maintain network capability anywhere, anytime without interruption on their ongoing sessions. 


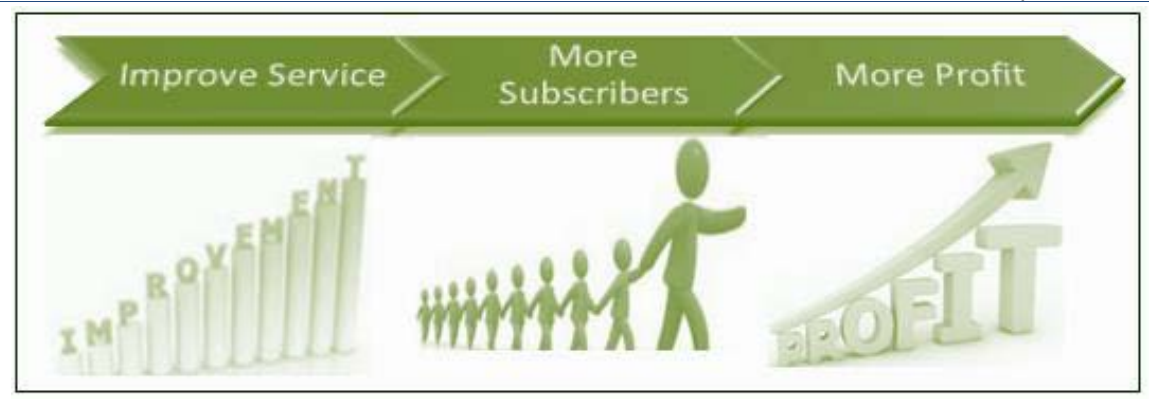

Figure 3. Operators' vsion of using heterogeneous wireless communication technologies communication technologies

\section{Why are heterogeneous wireless communication technologies necessary?}

3GPP and non-3GPP include multiple integrated mobile systems and wireless networks and all of them coexist in a heterogeneous wireless access environment. At the same time each RAT has its advantages and disadvantages. Therefore, the complementarity between RATs is still required due to their characteristics. For example, the integration between WIMAX and LTE would satisfy MUs' demands to ongoing their sessions without noticeable degradation. Consequently, it would allow the service provider to get more profit.

\section{What is the handover management within heterogeneous wireless communication technologies?}

Handover management is a process which allows the MUs to continue their ongoing sessions when moving within the same RAT coverage areas or traversing different RATs. In heterogeneous wireless communication technologies, the handover management is crucial because RATs typically differ in terms of multiple parameters such as RSS, data rate, reliability, service cost, security, power consumption requirements, coverage area and latency. Therefore, complementarity to these RATs through VHO interworking architectures is essential to provide ubiquitous wireless access ability with the best available access network which suits the MU's requirements (e.g., high coverage area, high data rate and low cost).

\section{Classifications for VHO Approaches of 3GPP and non-3GPP}

This section presents VHO approaches of 3GPP and non-3GPP proposed in the literature and classifies them into two categories based on $4 G$ and $5 G$ for which their characteristics have been discussed. We identify the two categories as: (A) 4G based category which includes $4 G$ and/or the rest of 3GPP previous generation mobile systems (2G-3G) and/or non-3GPP wireless networks. (B) $5 \mathrm{G}$ based category which includes $5 \mathrm{G}$ and/or the rest of 3GPP previous generation mobile systems (2G-4G) and/or non-3GPP wireless networks.

\subsection{G Category}

In this category, plenty of VHO approaches have been proposed in the literature. In [29], [30] and [31], seventeen, fifteen and ninety nine VHO approaches have been surveyed, respectively. It has been noticed in [29-31] that the VHO approaches are mostly in the practical where the evolution

methods in these surveys are various between real environment, testbed, simulation experiment and analytical modeling. 


\subsection{G Category}

Although one of the most important goals of $5 \mathrm{G}$ is to provide ubiquitous wireless access abilities [15], it would not be perfectly able to achieve the goal without cooperating with the rest of wireless communication technologies of 3GPP and non-3GPP. To the best of my knowledge the current research works of this category are mainly confined in $5 \mathrm{G}$ where the recent overview of VHO in $4 \mathrm{G}$ and $5 \mathrm{G}$ has showed that in [32].

\section{Comparison for VHO Approaches of 3GPP and non-3GPP}

Section III has presented two categories of VHO approaches based on 4 G and 5 G for which their characteristics have been discussed. 4G Category has substantially presented and evaluated its VHO approaches using various evaluations methods. As the final success of $5 \mathrm{G}$ will depend upon when it is fully implemented and the new services and contents made available to MUs, it would be preferable to design and develop scenarios of $5 \mathrm{G}$ Category for evaluating real-world deployments, testbed, simulation experiment and analytical modeling compared with the huge number of previous works in $4 \mathrm{G}$ Category.

\section{Conclusion}

This paper has presented a study for VHO approaches of 3GPP and non-3GPP proposed in the literature and classified them into two categories based on $4 G$ and $5 G$ for which their characteristics have been discussed. It has been concluded that the $5 G$ Category should be an active area of research compared with 4G Category which has obviously succeeded in presenting and evaluating plenty of VHO approaches.

\section{REFERENCES}

[1] Haji, A.; Ben Letaifa, A.; Tabbane, S.; "Integration of WLAN, UMTS and WiMAX in 4G," 16th International Conference Electronics, Circuits, and Systems 2009 (ICECS 2009), 13-16 Dec 2009, pp. 307-310.

[2] Torad, M.; El Qassas, A.; Al Henawi, H.; "Comparison between LTE and WiMAX Based on System Level Simulation Using OPNET Modeler (Release 16)," 28th National Radio Science Conference 2011 (NRSC 2011), 26-28 Apr 2011, pp. 1-9.

[3] Guifen, G.; Guili, P.;, "The Survey of GSM Wireless Communication System," International Conference on Computer and Information Application 2010 (ICCIA 2010), 3-5 Dec 2010, pp. 121-124.

[4] Kazemi, R.; Mosayebi, R.; Etemadi, S.M.; Boloursaz, M.; Behnia, F.;, "A Lower Capacity Bound of Secure End to End Data Transmission via GSM Network," 6th International Symposium on Telecommunications 2012 (IST 2012), 6-8 Nov 2012, pp. 1015-1020.

[5] Tae, S.K., Soo, W.K.; " "A Method for Reducing a Bumblebee Noise Generated by a GSM Technology in a Smartphone," 12th International Conference on Intelligent Systems Design and Applications 2012 (ISDA 2012), 27-29 Nov 2012, pp. 927-930.

[6] Nkansah-Gyekye, Y.; Agbinya, J.I.;, “A Vertical Handoff Decision Algorithm for Next Generation Wireless Networks," 3rd International Conference on Broadband Communications, Information Technology \& Biomedical Applications, 23-26 Nov 2008, pp. 358-364. 
Dr Omar Khattab; 3GPP Generation Mobile Systems and Non-3GPP Wireless Networks: A New Class of Octagon-Cell Interconnected Networks, Transactions on Networks and Communications, Volume 6 No. 2, April (2018); pp: 1-9

[7] Yi, B.L.; Yieh, R.H.; Yuan, KC.; Imrich, C.;, "Mobility Management: from GPRS to UMTS," Wireless Communications and Mobile Computing, vol. 1, no. 4, 2001, pp. 339-359.

[8] Darnbrough, J.;, "Market Aspects of UMTS," IEE Colloquium on Personal Communications in the 21st Century (I), 5 Feb 1998, pp. 1-26.

[9] Rabbani, M.G.; Kamruzzaman, J.; Gondal, I.; Ahmad, I.;, “A new Resource Distribution Model for Improved QoS in an Integrated WiMAX/WiFi Architecture," 7th International Wireless Communications and Mobile Computing Conference 2011 (IWCMC 2011), 4-8 Jul 2011, pp. 266-271.

[10] Datar, R.V.;, "WiFi and WiMAX-Break through in Wireless Access Technologies," IET International Conference on Wireless, Mobile and Multimedia Networks, 11-12 Jan 2008, pp. 141-145.

[11] David, M.S.; Jose, F.M.; Jorge, C.P.; Daniel, C.; Salvador, G.; Narc'ıs, C.; "On the Way Towards FourthGeneration Mobile:3GPP LTE and LTE-Advanced," Journal on Wireless Communications and Networking, vol. 2009, no. 4, 2009, pp. 1-10.

[12] Sengar, S.S.; Tyagi, N.; Singh, A.P.;, "A Survey on WiMAX-3G interworking," 3rd International Conferenceon Communication Software and Networks 2011 (ICCSN 2011), 27-29 May 2011, pp. 54-58.

[13] Ahmadi, S.;, "An Overview of Next-Generation Mobile WiMAX Technology," IEEE Communication Magazine, vol. 47, no. 6, Jun 2009, pp. 84-98.

[14] Talukdar, A.; Mondal, B.; Cudak, M.; Ghosh, A.; Fan, Wang.; "Streaming Video Capacity Comparisons of Multi-Antenna LTE Systems," 71st Vehicular Technology Conference 2010 (VTC 2010-Spring), 16-19 May 2010, pp. 1-5.

[15] Abdullah M.; Sultan, A.; Hassan, A.;, "4G and 5G Mobile Communication Networks: Features Analysis, Comparison and Proposed Architecture," International Journal of Computer Science and Technology, vol. 7, no. 2, Jun 2016, pp. 154-160.

[16] Arun, A.; Gourav, M.; Kabita A.;, "The 5th Generation Mobile Wireless Networks- Key Concepts, Network Architecture and Challenges," American Journal of Electrical and Electronic Engineering, vol. 3, no. 2, Mar 2015, pp. 22-28.

[17] 4G LTE World Coverage Map. (2017). WorldTimeZone. Retrieved 16 Apr, 2017, from https://www.worldtimezone.com/4g.html.

[18] 5G Field Testing, Trials, Research, Development World Coverage Map. (2017). WorldTimeZone. Retrieved 16 Apr, 2017, from https://www.worldtimezone.com/5g.html.

[19] Akkari, N.; Tohme, S.;, "Introducing Intelligent Vertical Handover in Next Generation Networks," International Conference Signal Processing and Communications 2007 (ICSPC 2007), 24-27 Nov 2007, pp. 728-731.

[20] Chang, J.M.; Abichar, Z.; Chau-Yun, H.;, "WiMAX or LTE: Who Will Lead the Broadband Mobile Internet?," IT Professional, vol. 12, no. 3, May-Jun 2010, pp. 26-32. 
[21] Seddigh, N.; Nandy, B.; Makkar, R.; Beaumont, J.F.;, "Security Advances and Challenges in 4G Wireless Networks," 8th Annual International Conference on Privacy Security and Trust 2010 (PST 2010), 17-19 Aug 2010, pp. 62-71.

[22] 3GPP TR 25.814 v7.1.0, “Physical Layer Aspects for Evolved Universal Terrestrial Radio Access (UTRA) Release 7".

[23] Khan, M.M.A.; Ismail, M.F.; Dimyati, K.;, "Seamless Handover between WiMAX and UMTS," 9th Malaysia International Conference on Communications 2009 (MICC 2009), 15-17 Dec 2009 pp. 826-830.

[24] Alkhayat, I.; Kumar, A.; Elmaghraby, A.;, "Seamless Connectivity Scheme for Heterogeneous Wireless Networks," International Symposium on Signal Processing and Information Technology 2008 (ISSPIT 2008), 16-19 Dec 2008, pp. 363-368.

[25] Ben-Jye, C.; Jun-Fu, C.;, "Cross-Layer-Based Adaptive Vertical Handoff With Predictive RSS in Heterogeneous Wireless Networks," IEEE Transactions on Vehicular Technology, vol. 57, no. 6, Nov 2008, pp. 3679-3692.

[26] [26] The Mobile Broadband Standard. (Jun/2013). 3rd Generation Partnership Project (3GPP). Retrieved 27 May, 2014, from http://www.3gpp.org/technologies/keywords-acronyms/97-Ite-advanced.

[27] Fangmin, X.; Luyong, Z.; Zheng, Z.;, "Interworking of Wimax and 3GPP Networks Based on IMS [IP Multimedia Systems (IMS) Infrastructure and Services]," IEEE Communications Magazine, vol. 45, no. 3, Mar 2007, pp. 144-150.

[28] Angoma, B.; Erradi, M.; Benkaouz, Y.; Berqia, A.; Akalay, M.C.;, "HaVe-2W3G: A Vertical Handoff Solution between WLAN, WiMAX and 3G Networks," 7th International Wireless Communications and Mobile Computing Conference 2011 (IWCMC 2011), 4-8 Jul 2011, pp. 101-106.

[29] 9] Khattab, O.; Alani, O.;, "A Survey on Media Independent Handover (MIH) and IP Multimedia Subsystem (IMS) in Heterogeneous Wireless Networks," International Journal of Wireless Information Networks (IJWIN), Springer, vol. 20, no. 2, Jun 2013, pp. 215-228.

[30] Khattab, O.; Alani, O.;, "A Survey on MIH vs. ANDSF: Who Will Lead the Seamless Vertical Handover Through Heterogeneous Networks?," International Journal of Future Generation Communication and Networking (IJFGCN), vol. 6, no. 4, Aug 2013, pp. 1-11.

[31] Ahmed, A.; Boulahia, L.; Gaiti, D.;, "Enabling Vertical Handover Decisions in Heterogeneous Wireless Networks: A State-of-the-Art and A Classification," IEEE Communications Surveys \& Tutorials, vol. PP, no. 99, 2013, pp. 1-36.

[32] Feras, Z.; Suhaidi, H.; Adib, H.;, "Vertical Handover in Wireless Heterogeneous Networks," Journal of Telecommunication, Electronic and Computer Engineering, vol. 9, no. 1-2 , 2017, pp. 81-85. 